\title{
ADSORÇÃO DE CORANTES AZO PONCEAU EM SÍLICA GEL MODIFICADA COM APTES
}

\author{
A. V. de LIMA ${ }^{1}$, A. A. VIANA ${ }^{2}$, P. A. L. MOTA ${ }^{1}$, \\ A. B. F. VIEIRA ${ }^{1}$, M. R. de SOUZA ${ }^{1}$, N. G. NOGUEIRA ${ }^{1}$, L. A. de $\mathrm{SA}^{1}$, A. F. dos \\ SANTOS ${ }^{1}$ \\ R. S. ARAÚJO' ${ }^{1}$, F. M. T. de LUNA ${ }^{3}$, H. L. B. BUARQUE ${ }^{1,2}$ \\ ${ }^{1}$ Instituto Federal de Educação, Ciência e Tecnologia do Ceará, Campus Fortaleza, \\ Departamento de Química e Meio Ambiente \\ ${ }^{2}$ Instituto Federal de Educação, Ciência e Tecnologia do Ceará, Campus Fortaleza, \\ Programa de Pós-Graduação em Tecnologia e Gestão Ambiental \\ ${ }^{3}$ Universidade Federal do Ceará, Departamento de Engenharia Química \\ E-mail para contato: hbuarque@ifce.edu.br
}

\begin{abstract}
RESUMO - Os corantes da família Ponceau são utilizados em diversas indústrias têxteis e alimentícias e, consequentemente, devem ser removidos de águas residuais industriais. Este estudo avaliou a utilização de sílica gel comercial modificada e com um aminosilano na remoção por adsorção dos corantes Acid Red 18 e Acid Red 66. A modificação da sílica gel foi confirmada e uma otimização do $\mathrm{pH}$ dos ensaios adsortivos foi realizada. Dados de cinética e de equilíbrio de adsorção foram determinados pelo método da imersão, a $30^{\circ} \mathrm{C}, \mathrm{pH} \mathrm{3,0}$ e agitação de $160 \mathrm{rpm}$. Ainda, modelos cinéticos de pseudo-primeira ordem e pseudo-segunda ordem e isotermas de equilíbrio de Langmuir e de Langmuir-Freundlich (LF) foram ajustados aos dados. Os resultados demonstraram que a sílica gel modificada neste estudo se mostra um adsorvente promissor na remoção desses corantes, apresentando elevadas cinéticas, que podem ser bem representadas pelo modelo PSO. O modelo LF se ajustou melhor aos dados de equilíbrio.
\end{abstract}

\section{INTRODUÇÃO}

Anualmente, são produzidos cerca de um milhão de toneladas de uma grande variedade de diferentes corantes em todo o mundo (TUNÇ et al., 2012). Os principais corantes têxteis podem ser enquadrados em duas classes: azo e indigoide. Particularmente, a classe azo se caracteriza pela presença da ligação dupla entre nitrogênios na sua estrutura (MANU, CHAUDHARI, 2003).

Corantes sintéticos são usados de forma extensiva nas indústrias têxtil, alimentícia, papel, plásticos e couro por apresentarem melhor estabilidade e maior variedade quando comparados aos corantes naturais. Assim, tais indústrias produzem águas residuais altamente coloridas, as quais, se não adequadamente tratadas, podem causar impactos deletérios nos corpos aquáticos em que são lançadas (MALIK et al, 2007; SHARMA et al, 2011; SURESH et al., 2011). 
Destaque-se que os corantes do tipo azo constituem a classe de corante mais usada industrialmente, correspondendo a mais da metade dos corantes comercialmente utilizados (FAHMI et al., 2010; FRANCISCON et al., 2012; YIĞITOĞLU, TEMOÇIN, 2010). Ressalte-se que estes compostos são de difícil remoção por tratamentos convencionais em efluentes industriais (e.g., lodo ativado, coagulação e floculação), a despeito de poderem se tornar tóxicos, carcinogênicos e mutagênicos no ambiente (GUPTA, SUHAS, 2009; KODAM, GAWAI, 2006; YIĞITOĞLU, TEMOÇIN, 2010).

Nos últimos anos, alguns estudos têm mostrado que adsorventes comerciais de baixo custo (e.g., sílica gel) modificados superficialmente podem ser eficientes na remoção de contaminantes ambientais (ANDRZEJEWSKA et al., 2007; CESTARI et al., 2009; DONIA et al., 2009; KUSHWAHA et al., 2010; MAHMOODI et al., 2011; XIA et al., 2011; YIĞITOĞLU, TEMOÇIN, 2010). Assim, adsorventes silicosos superficialmente funcionalizados com diferentes grupos funcionais ativos são de particular interesse. Estes materiais são capazes de interagir com diversas substâncias orgânicas quando é enxertada com compostos quelantes, fazendo-o um adsorvente promissor para adsorção seletiva de poluentes.

Neste contexto, este estudo objetivou avaliar a utilização de uma sílica gel comercial modificada superficialmente com o silanizante 3-aminopropiltrietoxisilano (APTES) na remoção de dois compostos azoicos: o Ponceau 4R (acid red 18) e o Ponceau BS (acid red 66), comumente utilizados como corante têxtil e corante alimentício, respectivamente. Dados de equilíbrio e de cinética de adsorção foram determinados, em $\mathrm{pH}$ ácido e temperatura ambiente, e feitos ajustar modelos cinéticos e isotermas teóricas. Ainda, alguns dados de caracterização do adsorvente usado e da otimização da faixa de $\mathrm{pH}$ ideal para o processo também foram obtidos.

\section{METODOLOGIA}

Soluções estoques de $2000 \mathrm{mg} / \mathrm{L}$ dos corantes Ponceau 4R (C.I. 16255; 604,47 g/mol) e Ponceau BS (C.I. 26905; 556,48 g/mol), marca Merck, ajustadas para pH iguala a 3,0, foram preparadas para uso nos ensaios de adsorção.

A determinação das concentrações das soluções dos corantes nos ensaios de adsorção foram realizadas em espectrofotômetro de absorção UV/VIS (marca Shimatzu, modelo UV 1601PC), no comprimento de onda de $510 \mathrm{~nm}$ para o acid red 18 e $505 \mathrm{~nm}$ para o acid red 66, após tamponamento para pH 7,0.

Uma sílica gel comercial (SGC), marca VETEC, macerada, peneirada (75 a $150 \mu \mathrm{m}$ ) e funcionalizada com APTES, marca Sigma-Aldrich, segundo o procedimento de Donia et al. (2009), foi avaliada como adsorvente neste estudo.

A funcionalização da sílica gel modificada (SGM) foi confirmada por espectrometria de infravermelho (FTIR), realizada em espectrômetro da marca Shimadzu, modelo IRAffinity-1. Ensaios de ponto de carga zero (PCZ) também foram realizados para a SGM, seguindo o procedimento de GUILARDUCI et al. (2006), de modo a estimar o pH de mudança da carga superficial do adsorvente. 
Ainda, antercedendo aos ensaios cinéticos e de equilíbrio de adsorção, foram avaliados os valores de $\mathrm{pH}$ ótimos para posteriores ensaios, nos dois corantes, numa faixa de $\mathrm{pH}$ de 3,0 a 9,0 e de concentrações de $30 \mu \mathrm{mol} / \mathrm{L}(18 \mathrm{mg} / \mathrm{L})$ a $120 \mu \mathrm{mol} / \mathrm{L}(72 \mathrm{mg} / \mathrm{L})$. Utilizou-se da técnica de delineamento composto central rotacional (DCCR) com quatro pontos axiais e quatro pontos centrais e da análise de curvas de superfícies para tal avaliação (RODRIGUES, EMMA, 2005). Os valores de $\mathrm{pH}$ e de concentração inicial do adsortivo (fatores) usados neste delineamento são mostrados na Tabela 1. A variável resposta utilizada foi a quantidade adsorvida nos testes.

Tabela 1 - Planejamento de experimentos para avaliação da faixa ótima de $\mathrm{pH}$.

\begin{tabular}{ccc|ccc|ccc}
\hline ENSAIO & $\mathbf{p H}$ & $\begin{array}{c}\text { Co } \\
(\boldsymbol{\mu m o l} / \mathbf{L})\end{array}$ & ENSAIO & $\mathbf{p H}$ & $\begin{array}{c}\text { Co } \\
(\boldsymbol{\mu m o l} / \mathbf{L})\end{array}$ & ENSAIO & $\mathbf{p H}$ & $\begin{array}{c}\mathbf{C o} \\
(\boldsymbol{\mu m o l} / \mathbf{L})\end{array}$ \\
\hline 1 & 6,0 & 138,6 & 5 & 1,8 & 75,0 & 9 & 3,0 & 30,0 \\
2 & 6,0 & 11,4 & 6 & 6,0 & 75,0 & 10 & 9,0 & 30,0 \\
3 & 3,0 & 120,0 & 7 & 6,0 & 75,0 & 11 & 9,0 & 120,0 \\
4 & 10,2 & 75,0 & 8 & 6,0 & 75,0 & 12 & 6,0 & 75,0 \\
\hline
\end{tabular}

Os ensaios cinéticos foram realizados para cada corante em meio ácido ( $\mathrm{pH} 3$ ), previamente avaliado como o melhor meio para o processo, e sob temperatura de $30^{\circ} \mathrm{C}$, seguindo a metodologia da imersão em banho finito, conforme descrita em Rouquerol et al. (1999).

Todos estes ensaios cinéticos foram conduzidos em células constituídas por erlenmeyers de $125 \mathrm{~mL}$, no qual eram adicionados inicialmente $20 \mathrm{~mL}$ de água destilada, aproximadamente $0,20 \mathrm{~g}$ da SGM e, posteriormente, mais $20 \mathrm{~mL}$ de soluções aquosas ácidas de 100 e de $200 \mathrm{mg} / \mathrm{L}$ dos corantes Ponceau 4R e Ponceau BS, tornando-as com concentrações iniciais de 50 e de $100 \mathrm{mg} / \mathrm{L}$, respectivamente. Os erlenmeyers foram agitados a $160 \mathrm{rpm}$ numa incubadora com agitação orbital, marca Marconi modelo MA 410/CF ao longo de 48 horas. Então, em tempos pré-definidos, alíquotas de $7 \mathrm{~mL}$ eram retiradas das células, tamponadas para $\mathrm{pH}$ 7,0, centrifugadas e analisadas espectrofotometricamente.

Os dados cinéticos obtidos foram feitos ajustar os modelos de pseudo-primeira ordem (PPO) e pseudo-segunda ordem (PSO), expressos na Equação (1) e na Equação (2), respectivamente. Os coeficientes de determinação e os desvios relativos médios para os ajustes foram calculados.

$$
\begin{aligned}
& \frac{\mathrm{dq}_{\mathrm{t}}}{\mathrm{dt}}=\mathrm{k}_{1}\left(\mathrm{~m}_{1}-\mathrm{q}_{\mathrm{t}}\right), \\
& \frac{\mathrm{dq}_{\mathrm{t}}}{\mathrm{dt}}=\mathrm{k}_{2}\left(\mathrm{~m}_{2}-\mathrm{q}_{\mathrm{t}}\right)^{2},
\end{aligned}
$$

em que $\mathrm{q}_{\mathrm{t}}$ é a quantidade adsorvida num instante $\mathrm{t}, \mathrm{k}_{1}$ e $\mathrm{m}_{1}$, e $\mathrm{k}_{2}$ e $\mathrm{m}_{2}$ são os parâmetros dos modelos.

Os ensaios de equilíbrio também foram realizados em $\mathrm{pH} 3$ e temperatura de $30^{\circ} \mathrm{C}$, conduzidos nas mesmas células utilizadas no ensaio de cinética, seguindo a metodologia da imersão em banho finito. Foram adicionadas alíquotas de $20 \mathrm{~mL}$ de solução de corante com concentrações de $10 \mathrm{mg} / \mathrm{L}$ até $1200 \mathrm{mg} / \mathrm{L}$, no qual já estavam presentes $20 \mathrm{~mL}$ de água 
destilada e cerca de $0,2 \mathrm{~g}$ do adsorvente, resultando numa faixa de concentrações iniciais do adsortivo de 5 a $600 \mathrm{mg} / \mathrm{L}$. Os erlenmeyers foram submetidos a uma agitação nas mesmas condições e equipamento do ensaio cinético. A solução ficou em contato com o adsorvente por 48 horas de modo a garantir o equilíbrio. As alíquotas eram então retiradas das células, tamponadas em $\mathrm{pH}$ neutro, centrifugadas, e analisadas espectrofotometricamente.

Os dados de equilíbrio determinados foram feitos ajustar as isotermas de Langmuir (ML), de Freundlich (MF) e de Langmuir-Freundlich (MLF) representadas nas Equações (3), (4) e (5), respectivamente. Os coeficientes de determinação para os ajustes foram calculados.

$\mathrm{q}_{\mathrm{e}}=\frac{\mathrm{m}_{\mathrm{L}} \mathrm{k}_{\mathrm{L}} \mathrm{C}_{\mathrm{e}}}{1+\mathrm{k}_{\mathrm{L}} \mathrm{C}_{\mathrm{e}}}$

$\mathrm{q}_{\mathrm{e}}=\mathrm{k}_{\mathrm{F}} \mathrm{C}_{\mathrm{e}}^{1 / \mathrm{n}}$,

$\mathrm{q}_{\mathrm{e}}=\frac{\mathrm{m}_{\mathrm{L}} \mathrm{k}_{\mathrm{L}} \mathrm{C}_{\mathrm{e}}^{1 / \mathrm{n}}}{1+\mathrm{k}_{\mathrm{L}} \mathrm{C}_{\mathrm{e}}^{1 / \mathrm{n}}}$,

em que $\mathrm{q}_{\mathrm{e}}$ e $\mathrm{C}_{\mathrm{e}}$ são as quantidades de corante no adsorvato e na fase líquida no equilíbrio, $\mathrm{m}_{\mathrm{L}}, \mathrm{k}_{\mathrm{L}}, \mathrm{k}_{\mathrm{F}}$ e $\mathrm{n}$ são os parâmetros dos modelos.

\section{RESULTADOS E DISCUSSÃO}

A comparação dos espectros obtidos nos ensaios de FTIR, obtidos, nas mesmas condições, para a SGC antes e depois do procedimento de funcionalização (silanização), são mostrados na Figura 3 e confirmam a modificação da SGC utilizada, haja vista que se observa que as bandas características dos grupos silanóis e siloxanos $\left(3448 \mathrm{~cm}^{-1}, 1085 \mathrm{~cm}^{-1}\right.$, $954 \mathrm{~cm}^{-1}, 799 \mathrm{~cm}^{-1}$ ) diminuem consideravelmente, aliado ao surgimento de bandas características muito discretas de estiramento $=\mathrm{CH}_{2}$ entre $2850 \mathrm{~cm}^{-1}$ e $2950 \mathrm{~cm}^{-1}$, conforme também verificaram Donia et al. (2009).

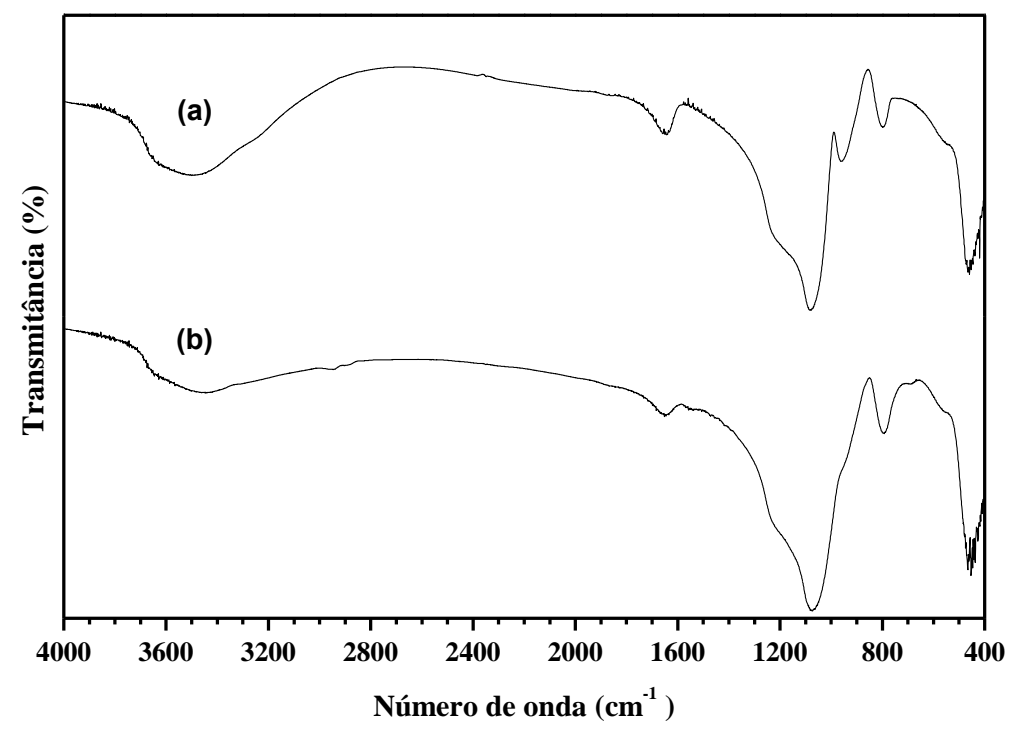

Figura 1 - Os espectros de FTIR da: (a) SGC; (b) SGM. 
A partir dos ensaios de PCZ indicaram uma mudança na carga superficial do adsorvente avaliado em $\mathrm{pH} 6,7$. Isto é, o adsorvente em meio aquoso ácido $(\mathrm{pH}<6,7)$ tem sua carga superficial líquida positiva, o que deve favorecer a adsorção de contaminantes orgânicos aniônicos, a exemplo de corantes azoicos contendo grupos sulfônicos. Contrariamente, em meio neutro a básico $(\mathrm{pH}>6,7)$, as cargas superficiais da SGM são predominantemente negativas, potencializando a adsorção de espécies catiônicas, tais como metais pesados. Complementarmente, as curvas de superfície de resposta obtidas nos ensaios de otimização do pH por DCCR, para o processo adsorção avaliado, indicam que as capacidades de adsorção dos corantes no adsorvente são incrementadas à medida que o pH do meio vai se tornando mais ácido, principalmente quando os valores são inferiores a 4,0.

Os ensaios cinéticos e de equilíbrio foram, então, conduzidos seguindo a metodologia já descrita, fornecendo os resultados apresentados, respectivamente, nas Figuras 2 e 3. Os melhores modelos cinéticos (PSO) e de isotermas (MLF) ajustados aos dados também estão apresentados nestas figuras.

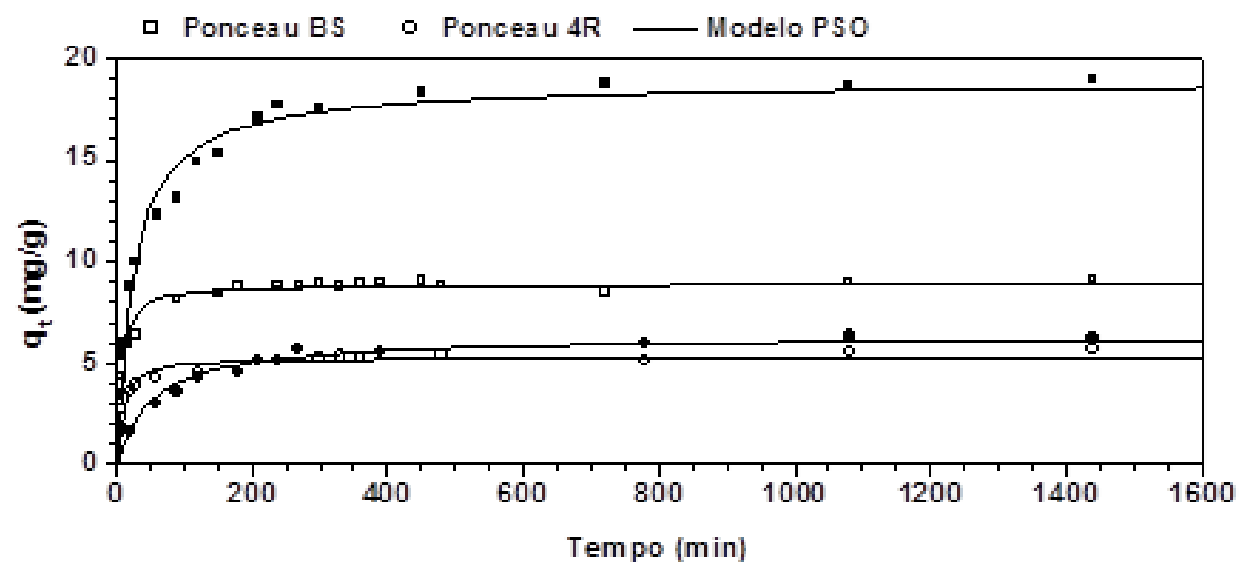

Figura 2 - Curvas cinéticas para a adsorção dos corantes estudados na SGM:

símbolos vazados são dados experimentais obtidos para concentração inicial de $50 \mathrm{mg} / \mathrm{L}$ e símbolos preenchidos são dados experimentais obtidos para concentração inicial de $100 \mathrm{mg} / \mathrm{L}$; linhas são modelos de PSO.

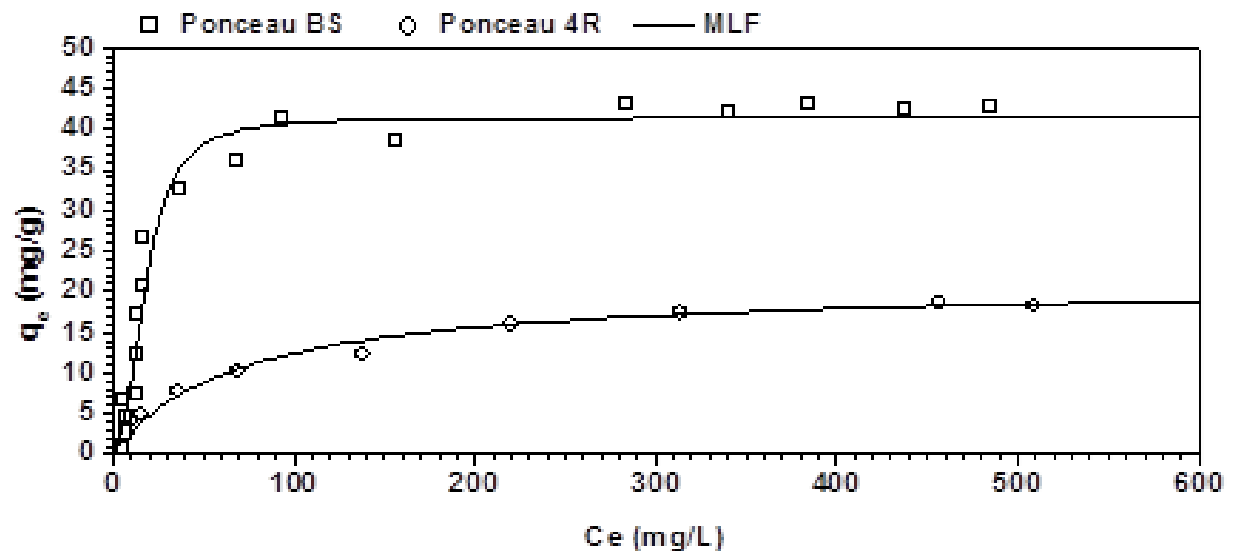

Figura 3 - Isotermas de equilíbrio da adsorção dos corantes estudados na SGM: símbolos são dados experimentais linhas são isotermas segundo o MLF. 
Ressalte-se que os parâmetros estimados no ajuste dos modelos cinéticos e de equilíbrio estão dispostos na Tabela 2 e na Tabela 3, respectivamente. Os coeficientes de determinação $\left(\mathrm{R}^{2}\right)$ obtidos no ajuste dos modelos aos dados experimentais também foram calculados e dispostos nestas tabelas.

Tabela 5 - Parâmetros dos modelos cinéticos ajustados na remoção dos corantes Ponceau $4 \mathrm{R}$ e Ponceau BS sobre a SGM a $30^{\circ} \mathrm{C}$.

\begin{tabular}{cccccccccc}
\hline Corante & $\begin{array}{c}\mathbf{C o} \\
(\mathbf{m g} / \mathbf{L})\end{array}$ & $\begin{array}{c}\boldsymbol{q}_{\boldsymbol{e} \mathbf{1}} \\
(\mathbf{m g} / \mathbf{g})\end{array}$ & $\begin{array}{c}\boldsymbol{k}_{\mathbf{1}} \\
\left(\mathbf{m i n}^{-\mathbf{1}}\right)\end{array}$ & $\mathbf{R}^{\mathbf{2}}$ & & $\begin{array}{c}\boldsymbol{q}_{\boldsymbol{e} \mathbf{2}} \\
(\mathbf{m g} / \mathbf{g})\end{array}$ & $\begin{array}{c}\boldsymbol{k}_{\mathbf{2}} \\
(\mathbf{g} / \mathbf{m g} \mathbf{m i n})\end{array}$ & $\mathbf{R}^{\mathbf{2}}$ \\
\hline \multirow{2}{*}{ Ponceau 4R } & 50 & 5,102 & 0,079 & 0,8528 & & 5,290 & 0,026 & 0,9254 \\
& 100 & 5,870 & 0,010 & 0,8900 & & 6,361 & 0,002 & 0,9240 \\
\hline \multirow{2}{*}{ Ponceau BS } & 50 & 8,672 & 0,087 & 0,8749 & & 8,892 & & 0,019 & 0,9391 \\
& 100 & 17,35 & 0,026 & 0,9076 & & 18,794 & & 0,002 & 0,9650 \\
\hline
\end{tabular}

Tabela 6 - Parâmetros dos modelos (ML, MF e MLF) ajustados aos dados experimentais de equilíbrio de adsorção dos corantes Ponceau 4R e Ponceau BS em SGM a $30^{\circ} \mathrm{C}$.

\begin{tabular}{|c|c|c|c|c|c|c|c|c|c|c|}
\hline \multirow[b]{2}{*}{ Corante } & \multicolumn{3}{|c|}{ ML } & \multicolumn{3}{|c|}{ MF } & \multicolumn{4}{|c|}{ MLF } \\
\hline & $\underset{(\mathrm{mg} / \mathrm{g})}{\mathbf{m}_{\mathbf{L}}}$ & $\begin{array}{c}\mathbf{K}_{\mathbf{L}} \\
(\mathbf{L} / \mathbf{m g})\end{array}$ & $\mathbf{R}^{2}$ & $\underset{(\mathbf{L} / \mathbf{m g})}{\mathbf{K}_{\mathbf{F}}}$ & $1 / n$ & $\mathbf{R}^{2}$ & $\underset{(\mathrm{mg} / \mathrm{g})}{\mathbf{m}_{\mathbf{L}}}$ & $\begin{array}{c}\mathbf{K}_{\mathbf{L}} \\
(\mathbf{L} / \mathbf{m g})\end{array}$ & $1 / n$ & $\mathbf{R}^{2}$ \\
\hline Ponc & 20,322 & 0,0158 & 0,986 & 1,6437 & 0,400 & 0,96 & 20,956 & 0,0145 & 1,000 & 0,9872 \\
\hline Ponceau BS & 47,129 & 0,0354 & 0,9221 & 6,7521 & 0,320 & 0,7884 & 41,388 & 0,0574 & 0,425 & 0,9645 \\
\hline
\end{tabular}

Os resultados revelam que o processo tem elevada cinética, particularmente em maiores concentrações iniciais, e que dentre os modelos cinéticos simplificados usados, o PSO representou muito bem os dados experimentais obtidos. Para os dados de equilíbrio, o MLF foi o modelo que melhor explicou a variação da quantidade adsorvida em termos da concentração de adsortivo, no equilíbrio.

Também se pode observar que a SGM adsorveu, no equilíbrio, maiores quantidades de Ponceau BS em relação ao Ponceau 4R, o que sugere que a presença do grupo azo adicional na estrutura molecular ressonante do Ponceau BS teve um efeito maior no fenômeno que a presença do grupo sulfônico no Ponceau 4R. Tais influências devem ser mais bem avaliadas em estudos posteriores com corantes com estruturas moleculares distintas.

\section{CONCLUSÕES}

A sílica gel funcionalizada com APTES neste estudo se mostrou um adsorvente promissor na remoção de corantes azo em meio ácido ( $\mathrm{pH}$ 3), resultando em capacidades de adsorção superiores a $20 \mathrm{mg} / \mathrm{g}$ e elevadas cinéticas. O processo pode ser bem representado por um modelo cinético de pseudo-segunda ordem e por um modelo de isoterma de equilíbrio de Langmuir-Freudlich. O corante com dois grupos azo na estrutura adsorveu mais que aquele com um único grupo. 


\section{AGRADECIMENTOS}

Os autores agradecem à Fundação Cearense de Apoio ao Desenvolvimento Científico e Tecnológico (FUNCAP), ao Conselho Nacional de Pesquisa e Desenvolvimento (CNPq) e ao Instituto Federal de Educação, Ciência e Tecnologia do Ceará (IFCE), pelas bolsas, de mestrado, de iniciação cientifica e de iniciação científica júnior, concedidas ao nosso grupo de pesquisa; e ao Laboratório de Processos Químicos e Ambientais e ao Laboratório de Tecnologia Química do Departamento de Química e Meio Ambiente do IFCE - Campus Fortaleza pelo suporte analítico e financeiro.

\section{REFERÊNCIAS}

ANDRZEJEWSKA, A., KRYSZTAFKIEWICZ, A., JESIONOWSKI, T. Treatment of textile dye wastewater using modified silica. Dyes Pigments, v. 75, p. 116-124, 2007.

CESTARI, A. R., VIEIRA, E. F. S., VIEIRA, G. S., COSTA, L. P., TAVARES, A. M. G., LOH, W., AIROLDI, C. The removal of reactive dyes from aqueous solutions using chemically modified mesoporous silica in the presence of anionic surfactant - The temperature dependence and a thermodynamic multivariate analysis. J. Hazard. Mater., v. 161, p. 307-316, 2009.

DONIA, A. M.; ATIA, A. A.; AL-AMRANI, W. A.; EL-NAHAS, A. M. Effect of Structural Properties of Acid Dyes on their Adsorption Behaviour from Aqueous Solutions by Amine Modified Silica. J. Hazard. Mater., v. 161, p. 1544-1550, 2009.

FAHMI, ARIFFIN, A., ARSHAD, S. M., ABIDIN, C. Z. A., RAHMAT, N. R. Decolourization and COD removal of Azo dye solution by repeated ozonation and biodegradation. In: BABY, S., SANDHU, P. S. (eds.) Water Sci. Technol., 2010, Bangkok, Thailand. Proceedings of the 2010 International Conference on Environmental Science and Technology, Bangkok, Research Publishing Services, 2010, p. 149-153.

FRANCISCON, E., GROSSMAN, M. J., PASCHOAL, J. A. R., REYES, F. G. R., DURRANT, L. R. Decolorization and biodegradation of reactive sulfonated azo dyes by a newly isolated Brevibacterium sp. strain VN-15. SpringerPlus, v. 1, n. 37, p. 1-10, 2012.

GUILARDUCI, V. V. S., MESQUITA, J. P., MARTELli, P. B., GORGULHO, H. F. Adsorção de fenol sobre carvão ativado em meio alcalino. Quim. Nova, v. 29, p. 1226-1232, 2006.

GUPTA, V. K., SUHAS. Application of low-cost adsorbents for dye removal - A review. J. Environ. Manage., v. 90, p. 2313-2342, 2009.

KODAM, K. M., GAWAI, K. R. Decolorisation of reactive red 11 and 152 azo dyes under aerobic conditions. Indian J. Biotechnol, v. 5, p. 422-424, 2006.

KUSHWAHA, A. K., GUPTA, N., CHATTOPADHYAYA, M. C. Enhanced adsorption of malachite green dye on chemically modified silica gel. J. Chem. Pharm. Res., v. 2, p. 34-45, 2010.

MAHMOODI, N. M., KHORRAMFAR, S., NAJAFI, F. Amine-functionalized silica nanoparticle: Preparation, characterization and anionic dye removal ability. Desalination, v. 279, p. 61-68, 2011. 
MALIK, R.; RAMTEKE, D. S., WATE, S. R. Adsorption of malachite green on groundnut shell waste based powdered activated carbon. Waste Manage., v. 27, p. 1129-1138, 2007.

MANU, B.; CHAUDHARI, S. Decolorization of indigo and azo dyes in semicontinuous reactors with long hydraulic retention time. Process Biochem., v. 38, p. 1213-1221, 2003.

RODRIGUES, M. I., EMMA, A. F. Planejamento de experimentos e otimização de processos. $1^{\text {a }}$ ed. Campinas: Casa do Pão Editora, 2005.

ROUQUEROL, F.; ROUQUEROL, J; SING, K. Adsorption by Powders \& Porous Solids: principles, methodology and applications. San Diego: Academic Press, 1999.

SHARMA, P., KAUR, H., SHARMA, M., SAHORE, V. A review on applicability of naturally available adsorbents for the removal of hazardous dyes from aqueous waste. Environ. Monit. Assess., v. 183, p. 151-195, 2011.

SURESH, S., SUGUMAR, R. W., MAIYALAGAN, T. Adsorption of Acid Red 18 from Aqueous Solution onto Activated Carbon Prepared from Murraya koenigii (Curry Tree) Seeds. Asian J. Chem., v. 23, p. 219-224, 2011.

TUNÇ, S.; GURKAN, T.; DUMAN, O. On-line spectrophotometric method for the determination of optimum operation parameters on the decolorization of Acid Red 66 and Direct Blue 71 from aqueous solution by Fenton process. Chem. Eng. J., v. 181, 'p. 431-442, 2012.

XIA, C., JING, Y., JIA, Y., YUE, D., MA, J., YIN, X. Adsorption properties of congo red from aqueous solution on modified hectorite: kinetic and thermodynamic studies.

Desalination, v. 265, p. 81-87, 2011.

YIĞITOĞLU, M., TEMOÇIN, Z. Removal of Benzidine-based Azo Dye from Aqueous Solution Using Amide and Amine-functionalized Poly(ethylene terephthalate) Fibers. Fiber. Polym., v. 11, p. 996-1002, 2010. 